\title{
Comparison of equations for the calculation of LDL-Cholesterol in hospitalized patients
}

\author{
J Martins $^{1}$, SAS Olorunju ${ }^{2}$, LM Murray ${ }^{1}$, TS Pillay ${ }^{1}$
}

\begin{abstract}
${ }^{1}$ Department of Chemical Pathology, University of Pretoria and NHLS Tshwane Academic Division, Faculty of Health Sciences, University of Pretoria and Steve Biko Academic Hospital, Pretoria, South Africa

${ }^{2}$ Medical Research Council, South Africa

Corresponding author: Tahir S. Pillay, Department of Chemical Pathology, Faculty of Health Sciences, University of Pretoria, Private Bag X323, Arcadia, Pretoria, South Africa.
\end{abstract}

Tel: +27-12-319-2155; Fax: +27-12-328-3600; Email: tspillay@ gmail.com

\section{Highlights}

-We evaluated the Friedewald Equation and three other recently developed formulae.

- The de Cordova, Chen \& Hattori formulae were used in patients with co-morbidities.

- More than 14000 lipid profiles were compared with direct measurement of LDL-C.

- The Hattori formula showed the best performance over all lipid values.

- Alternative formulae should be implemented in hospitalized patients.

\section{Abstract:}

Background: The Friedewald equation is widely used to calculate LDL-C for cardiovascular risk prediction but is less accurate with comorbidities and extreme lipid values. Several novel formulae have been reported to outperform the Friedewald formula.

Methods: We examined 14,219 lipid profiles and evaluated four formulae (Friedewald, Chen, de Cordova, Hattori) and compared these to direct measurement of LDL-C across various triglyceride (TG), total cholesterol (TC) and HDL-cholesterol (HDL-C) 
ranges using Beckman reagents and instruments. Linear regression and ROC analysis were performed.

Results: The de Cordova formula showed a high correlation with directly measured LDL-C $(r=0.90 p<0.001)$, comparable to the Friedewald calculated values for directly measured LDL-C $(r=0.95 p<0.001)$. The de Cordova formula was favorable in some ranges of HDL, TC and the lowest TG range $(r=0.97 p<0.001)$ but performed least well in comparison with the three other LDL-C calculations $(A U C=0.8331)$, demonstrating inconsistent bias. The Chen formula performed better than Friedewald (AUC=0.9049). The Hattori formula outperformed all formulae including Friedewald over various ranges of lipid values (AUC=0.9097).

Conclusions: We observe favorable correlations of the de Cordova formula with Friedewald at low TG values. However, the Hattori formula appears to be best for application in hospitalized patients, even at extreme lipid values.

Keywords: LDL-Cholesterol,Friedewald equation, hospitalized patients

\section{Graphical abstract}

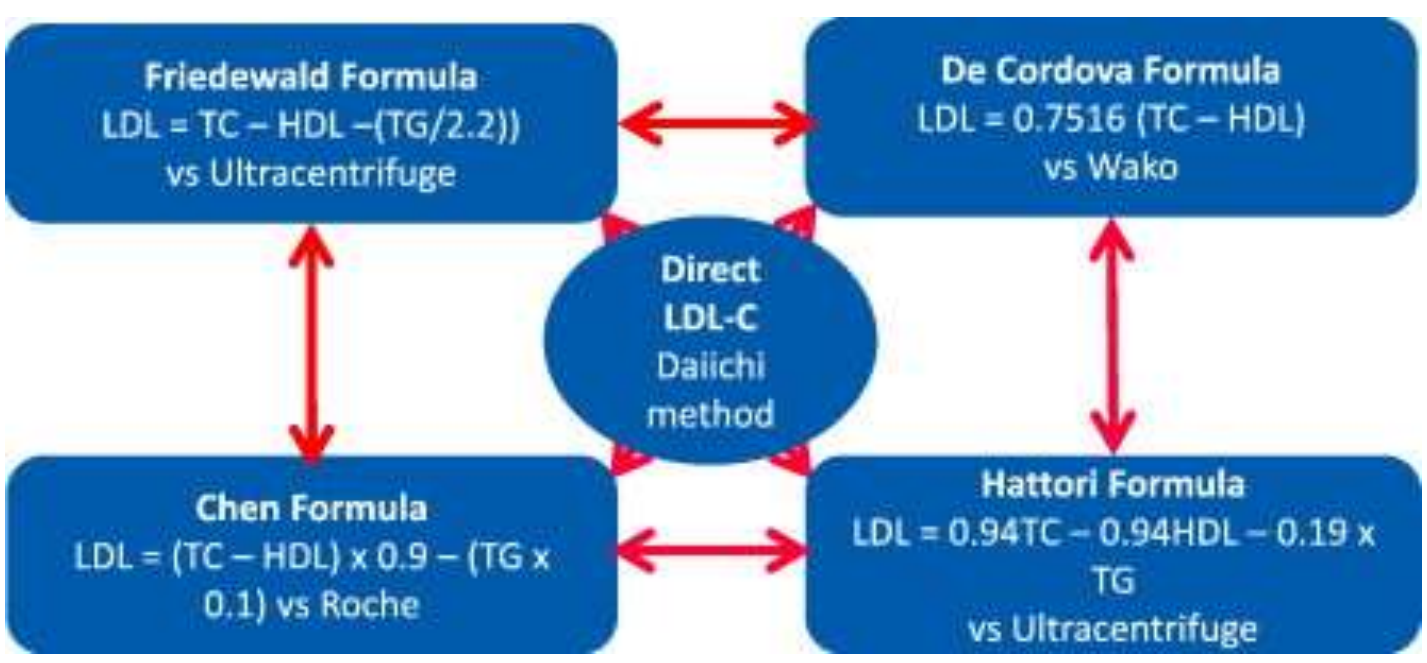




\section{Introduction}

LDL-cholesterol (LDL-C) is used for cardiovascular disease (CVD) risk assessment [1, 2]. The gold standard for measurement of LDL-C is by ultracentrifugation and betaquantification [3]. This is expensive and inconvenient for the routine laboratory. Other methods include direct measurement of LDL using a homogeneous assay, but this is too expensive for use in most laboratories. Furthermore, direct methods show poor performance with high triglyceride (TG) levels [4-6]. An earlier review comparing direct measurement of LDL-C vs calculation of LDL recommended the use of direct LDL measurements in hypertriglyceridaemic patients [6]. However, a recent study comparing eight direct measurements of LDL-C and HDL-C failed to show improved CVD risk classification of most direct methods over calculated LDL-C [4].

The first formula to calculate LDL-C was developed over 40 years ago by Friedewald[7]. The formula requires fasting plasma high density lipoproteincholesterol (HDL-C), total cholesterol (TC), and TG, and is calculated as LDL-C = TC - HDL - (TG/5) for $\mathrm{mg} / \mathrm{dl}(2.2 \mathrm{in} \mathrm{mmol} / \mathrm{l})$. This formula is less accurate in extremes of TG or TC values [7-10] or in patients with co-morbidities (eg. renal failure or diabetes) [2, 11], but is widely used. Several other formulae have been developed, but these did not perform better than Friedewald's calculation [12-14] or had varying results in different population groups $[10,15-19]$ and including those considering TG ratios [20, 21]. In the latest study validating a novel formula in comparison with Friedewald's calculation and the LDL-C reference method in 23055 patients, the benefits over Friedewald were not considered substantial enough to replace its use in clinical practice[22], demonstrating positive bias at low levels of LDL $(<1.81 \mathrm{mmol} / \mathrm{L})$. The previously published formula by de Cordova et al [23] has been reported to outperform several of the earlier LDL-C formulae, including Friedewald's formula, over a wide- 
range of lipid levels using the equation LDL-C $=0.7516$ (TC - HDL-C) in 10,664 Brazilian patients, including those with co-morbidities. However, this formula also showed bias at low levels of LDL-C in a subsequent study of 576 healthy subjects in South Africa [24].

As difficulties with LDL measurements prevail, a search for new formulae and emerging cardiovascular risk markers to improve accurate CVD prediction is ongoing. We validated the application of four formulae (Friedewald, de Cordova, Chen, Hattori) to calculate LDL-C in our population of hospitalized patients. We compared the formulae to the direct measurement of LDL-C, using the largest sample size to date, where multiple formulae are compared.

\section{Methods}

\subsection{Study population}

This was a retrospective evaluation of lipid profiles in 14, 219 patients in South Africa, from 1 January 2013 to 30 June 2013, using a database from the National Health Laboratory Services, the largest provider of laboratory services in South Africa. The laboratory is accredited by the South African National Accreditation System (SANAS), and serves a large tertiary academic hospital and surrounding clinics. The laboratory participates in the EQA program, the Thistle Lipid Programme. Procedures followed were approved by the Faculty of Health Sciences Research Ethics Committee of the University of Pretoria in accordance with the Helsinki Declaration.

Blood samples were collected into serum separator tubes to determine LDL-C, HDLC, TG and TC. Samples were centrifuged after collection and analysed immediately. Patient details were anonymized, with only patient age and gender reported. 
Measurements of LDL-C, HDL-C, TC and TG were performed using reagents by Beckman Coulter, according to the specification of the manufacturers using the Beckman DXC automated analyser (Brea, CA, USA).

The direct LDL-C method is a homogeneous assay without the need for any pretreatment or centrifugation steps and based on the Daiichi two-phase method [25]. The coefficient of variation (CV) of LDL-C using the homogenous method was $4.5 \%$ for level 1 and $4.0 \%$ for level 3.

The HDL-C measurement was performed using a homogenous, colorimetric, enzymatic method. The CV of the HDL level 1 and 3 respectively was $6.3 \%$ and 4.3\%. Total cholesterol measurement involved a colorimetric, enzymatic, timedendpoint method; the CV of the TC at level 1 and 3 were $3.4 \%$ and $4.6 \%$ respectively. Triglyceride measurement used a sequence of three coupled enzymatic steps to form a red quinoneimine dye. The CV of the TG measurements at level 1 and 3 were $4.3 \%$ and $3.9 \%$ respectively. The performance standards in terms of the CV for the lipid analysis were all within the acceptable CV for Beckman DxC800.

\subsection{Data Analysis}

Microsoft Excel was used to capture the data, according to the different lipid levels and for the calculation of LDL-C. STATA was used to perform the statistical analysis, which included a descriptive statistics summary. Pearson's correlation was performed for directly measured LDL-C and non-HDL-C, as well as between the four formula and directly measured LDL-C values obtained from the laboratory measurements. The root mean square error (rMSE) was calculated as a measure of accuracy in the differences between values predicted by an estimator and values observed from those being estimated to compare the formulae across various lipid ranges. Bland-Altman 
plots were used to evaluate the agreement between the four formulae and the directly measured LDL-C. ROC curve analysis was used to compare the performance of the different formulae considering the area under the curve (AUC). The coefficient of concordance was used to assess the relative performance of the different methods relative to the direct LDL-C measurement.

\section{Results}

A total of 14,219 lipid profiles were identified, of which $39 \%$ were male and $61 \%$ were female. Patient-specific data about presence/absence of disease, treatments and ethnicity was not available. The average age was 52 years with a range of directly measured LDL-C from 10.81 - $712.74 \mathrm{mg} / \mathrm{dl}$, mean $111.97 \mathrm{mg} / \mathrm{dl}[0.28-18.46 \mathrm{mmol} / \mathrm{l}$ (mean $2.9 \mathrm{mmol} / \mathrm{l} \pm 1.15$ Standard deviation (SD)]; for HDL-C from 4.63 - 400.39 $\mathrm{mg} / \mathrm{dl}$, mean $44.02 \mathrm{mg} / \mathrm{dl}$ [0.12 - $10.37 \mathrm{mmol} / \mathrm{l}$ (mean $1.14 \mathrm{mmol} / \mathrm{l} \pm 0.39 \mathrm{SD}$ )]; for TC from 9.28 - $1184.84 \mathrm{mg} / \mathrm{dl}$, mean $184.45 \mathrm{mg} / \mathrm{dl}[0.24-30.64 \mathrm{mmol} / \mathrm{l}$, (mean 4.77 $\mathrm{mmol} / \mathrm{l} \pm 1.47 \mathrm{SD})], \quad$ and $9.74-5837.91 \mathrm{mg} / \mathrm{dl}$, mean $162.10 \mathrm{mg} / \mathrm{dl}[0.11-65.91$ $\mathrm{mmol} / \mathrm{l}$ (mean $1.83 \mathrm{mmol} / \mathrm{l} \pm 1.90 \mathrm{SD}$ )] for TG. The mean (SD) calculated LDL-C values are shown in Table 1.

Table 1 Mean (SD) values of LDL-C and correlation with directly measured LDL-C per formula analyzed

\begin{tabular}{|c|c|c|c|c|}
\hline LDL-c & Formula & $\begin{array}{l}\text { Direct LDL } \\
\text { method }\end{array}$ & $\begin{array}{l}\text { Mean LDL-C (SD) } \\
\text { mg/dl (mmol/l) }\end{array}$ & $r \quad(\mathrm{P} \quad$ value $)$ \\
\hline Direct LDL-C & Directly measured & Daiichi & $112 \pm 45(2.9 \pm 1.15)$ & $1(<0.000)$ \\
\hline Friedewald & $\mathrm{LDL}=\mathrm{TC}-\mathrm{HDL}-(\mathrm{TG} / 2.2)$ & Ultracentrifuge & $108 \pm 48(2.8 \pm 1.24)$ & $0.9518(<0.000)$ \\
\hline De Cordova & $\mathrm{LDL}=0.7516(\mathrm{TC}-\mathrm{HDL})$ & Wako & $106 \pm 41(2.73 \pm 1.06)$ & $0.90(<0.000)$ \\
\hline Chen & $\mathrm{LDL}=(\mathrm{TC}-\mathrm{HDL}) \times 0.9-(\mathrm{TG} \times 0.1)$ & Roche & $119 \pm 46(3.08 \pm 1.19)$ & $0.9498(<0.000)$ \\
\hline Hattori & $\mathrm{LDL}=0.94 \mathrm{TC}-0.94 \mathrm{HDL}-0.19 \times \mathrm{TG}$ & Ultracentrifuge & $111 \pm 45(2.87 \pm 1.15)$ & $0.9626(<0.000)$ \\
\hline
\end{tabular}


Using Pearsons analysis, we show high correlations between the four formulae and directly measured LDL-C using the Daiichi two-phase method (Table 1 and Supplementary Figure 1). The de Cordova formula, although highly correlated with directly measured LDL-C $(r=0.90, P<0.001)$, was lower than the correlation observed with the other three formulae. The Friedewald formula had a higher correlation $(r=0.9518 P<0.001)$ than the Chen formula $(r=0.9498 P<0.001)$ but was lower than the correlation observed with the Hattori formula $(r=0.9626 \mathrm{P}<$ 0.001). Concordance correlations are shown as supplemental data (See Supplemental Figure 1).

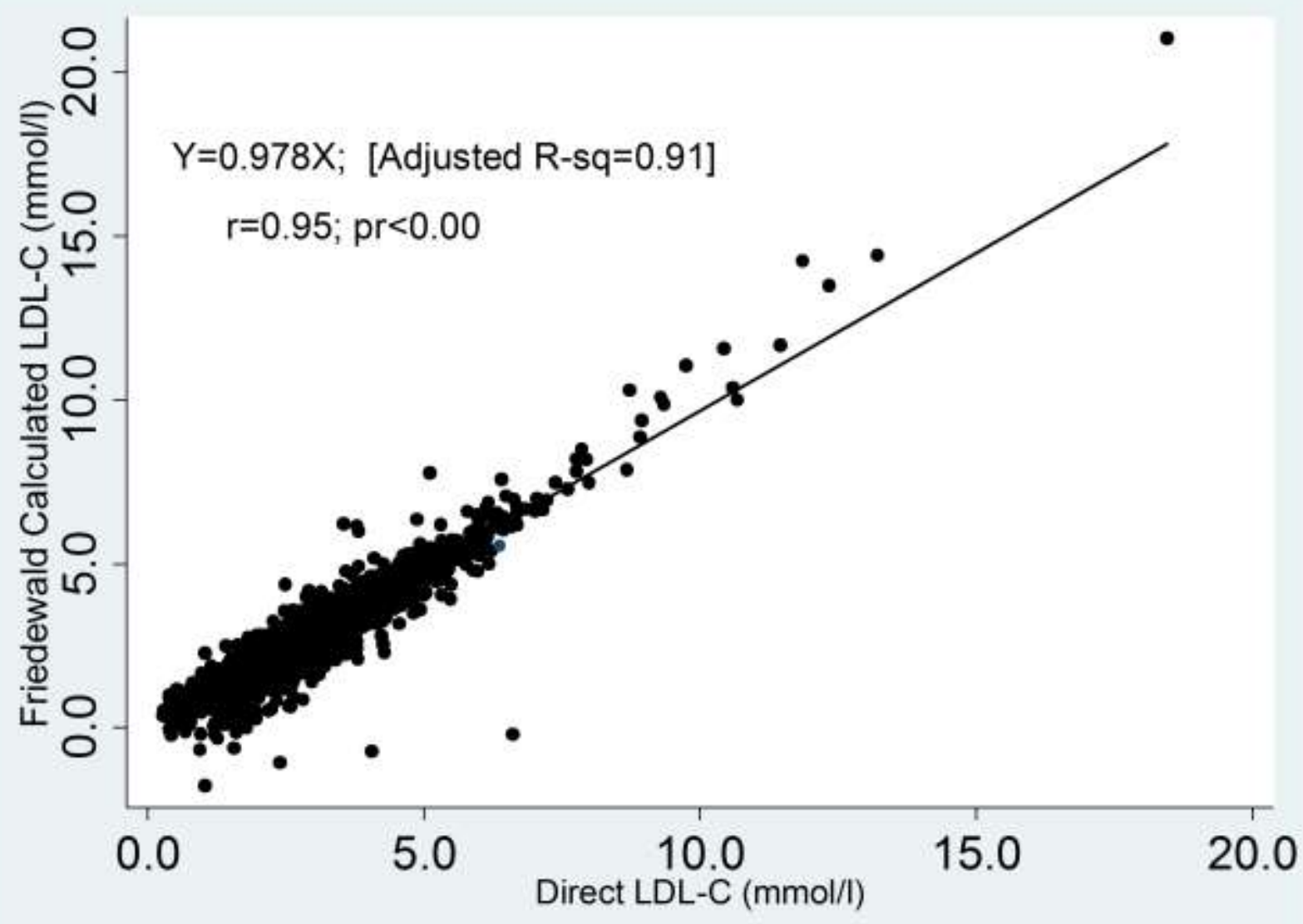

Figure 1 Correlation between the calculated LDL-C by the Hattori formula and directly measured LDL-C 
We also examined correlations between directly measured LDL-C and non-HDL-C (TC- HDL-C), LDL-C and TG, LDL-C and HDL-C/TG ratio, LDL-C and TC/HDL-C ratio, LDL-C and HDL-C/LDL-C ratio, and LDL-C and LDL-C/non-HDL-C ratio. Strong correlations were observed between LDL-C and non-HDL-C $(r=0.93)$ and TC and non-HDL-C $(r=0.964)$.

Using a ROC curve (Figure 2), the Hattori formula was shown to perform the best with an AUC of 0.9097 , followed by the Chen formula (AUC $=0.9049$ ), the Friedewald $(A U C=0.9018)$ and the de Cordova $(A U C=0.8331)$ formulae. Sensitivities and specificities are shown in Table 2.

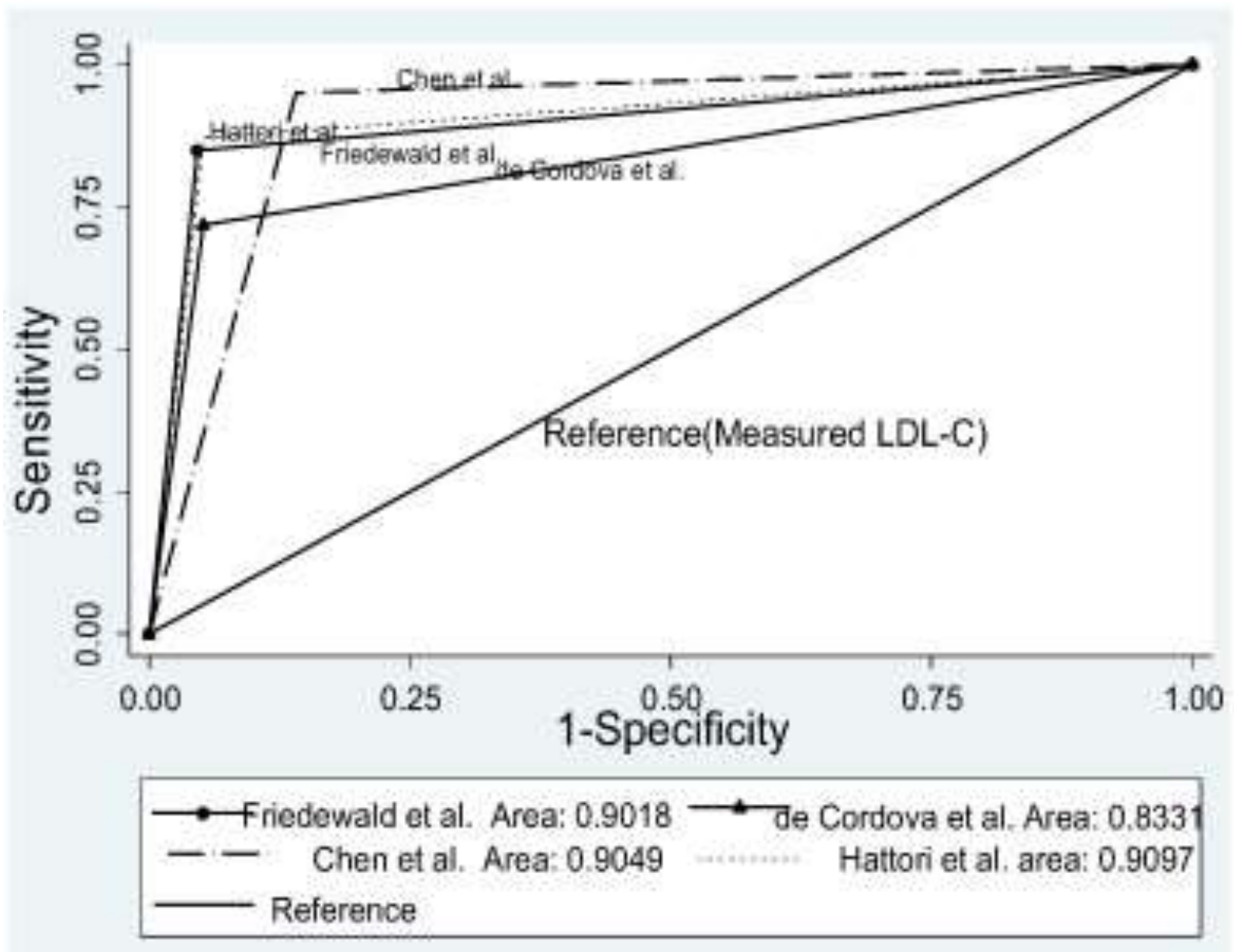

Figure 2 ROC curve of LDL-C calculated vs measured LDL-C for the Friedewald, de Cordova, Chen and Hattori formulae 
Table 2 Sensitivity, specificity and AUC for the Friedewald, Chen, de Cordova and Hattori formulae

\begin{tabular}{lcccc}
\hline Formula & Sensitivity (\%) & Specificity (\%) & AUC & (95\% Confidence Interval) \\
\hline Friedewald & 84.9 & 95.4 & 0.902 & $(95 \% \mathrm{Cl} 0.893,0.910)$ \\
Chen & 95 & 85.9 & 0.905 & $(95 \% \mathrm{Cl} 0.897,0.912)$ \\
De Cordova & 71.9 & 94.4 & 0.833 & $(95 \% \mathrm{Cl} 0.821,0.842)$ \\
Hattori & 87.1 & 94.8 & 0.910 & $(95 \% \mathrm{Cl} 0.902,0.918)$ \\
\hline
\end{tabular}

Table 3 demonstrates the rMSE of the four different formulae across different levels of HDL-C, TG and TC. The de Cordova formula was the least accurate at low HDL levels with a rMSE of 559 but at high HDL-C performed better (a rMSE of 102.7) than the Friedewald and Chen formulae with a rMSE of 130.2 and 106, respectively. The Hattori formula outperformed the other equations across all HDL-C and TG ranges, and TC ranges $73.10-218.87 \mathrm{mg} / \mathrm{dl}(1.89-5.66 \mathrm{mmol} / \mathrm{l})$. At TG $<187 \mathrm{mg} / \mathrm{dl}(<2.11$ $\mathrm{mmol} / \mathrm{l})$, the Hattori formula had a rMSE from 55.6 up to 85.9 with a rMSE of 280 for TG $>187 \mathrm{mg} / \mathrm{dl}(>2.11 \mathrm{mmol} / \mathrm{l})$, compared to an $\mathrm{rMSE}$ of $>400$ for the other three formulae. At the high end of TG ranges [ $>187 \mathrm{mg} / \mathrm{dl}(>2.11 \mathrm{mmol} / \mathrm{l})]$, the de Cordova showed the lowest accuracy (rMSE 479.6), followed by the Chen formula (a rMSE of 433.9) then the Friedewald formula (a rMSE of 418.5). At the lowest end of TG levels [17.71 $-90.35 \mathrm{mg} / \mathrm{dl}(0.20-1.02 \mathrm{mmol} / \mathrm{l})]$, the de Cordova formula had the highest accuracy with a rMSE of 54.2; the Friedewald formula had the lowest accuracy with a rMSE of 74.8. The Friedewald formula had the highest accuracy at the high end of TC ranges [250.20 - 522.82 mg/dl $(6.47-13.52 \mathrm{mmol} / \mathrm{l})$, with a rMSE of 120 . At the different TC ranges, the rMSE for the Friedewald formula was from 92.5 up to 316.9 , compared with 130.1 - 272 for the Chen formula, 163.1 - 323.7 with de Cordova and 82 - 185.6 with the Hattori formula. 
Figure 3 shows Bland-Altman difference plots of the directly measured LDL-C and the LDL-C derived from the four formulae. The mean bias for the Friedewald formula was $4.10 \pm 27.84 \mathrm{mg} / \mathrm{dl}(0.106 \pm 0.72 \mathrm{mmol} / \mathrm{l}), 6.73 \pm 37.51 \mathrm{mg} / \mathrm{dl}(0.174 \pm 0.97 \mathrm{mmol} / \mathrm{l})$ using the de Cordova formula, $-6.57 \pm 27.84 \mathrm{mg} / \mathrm{dl}(-0.17 \pm 0.72 \mathrm{mmol} / \mathrm{l})$ for the Chen formula, and $1.39 \pm 23.98 \mathrm{mg} / \mathrm{dl}(0.036 \pm 0.62 \mathrm{mmol} / \mathrm{l})$ for the Hattori formula

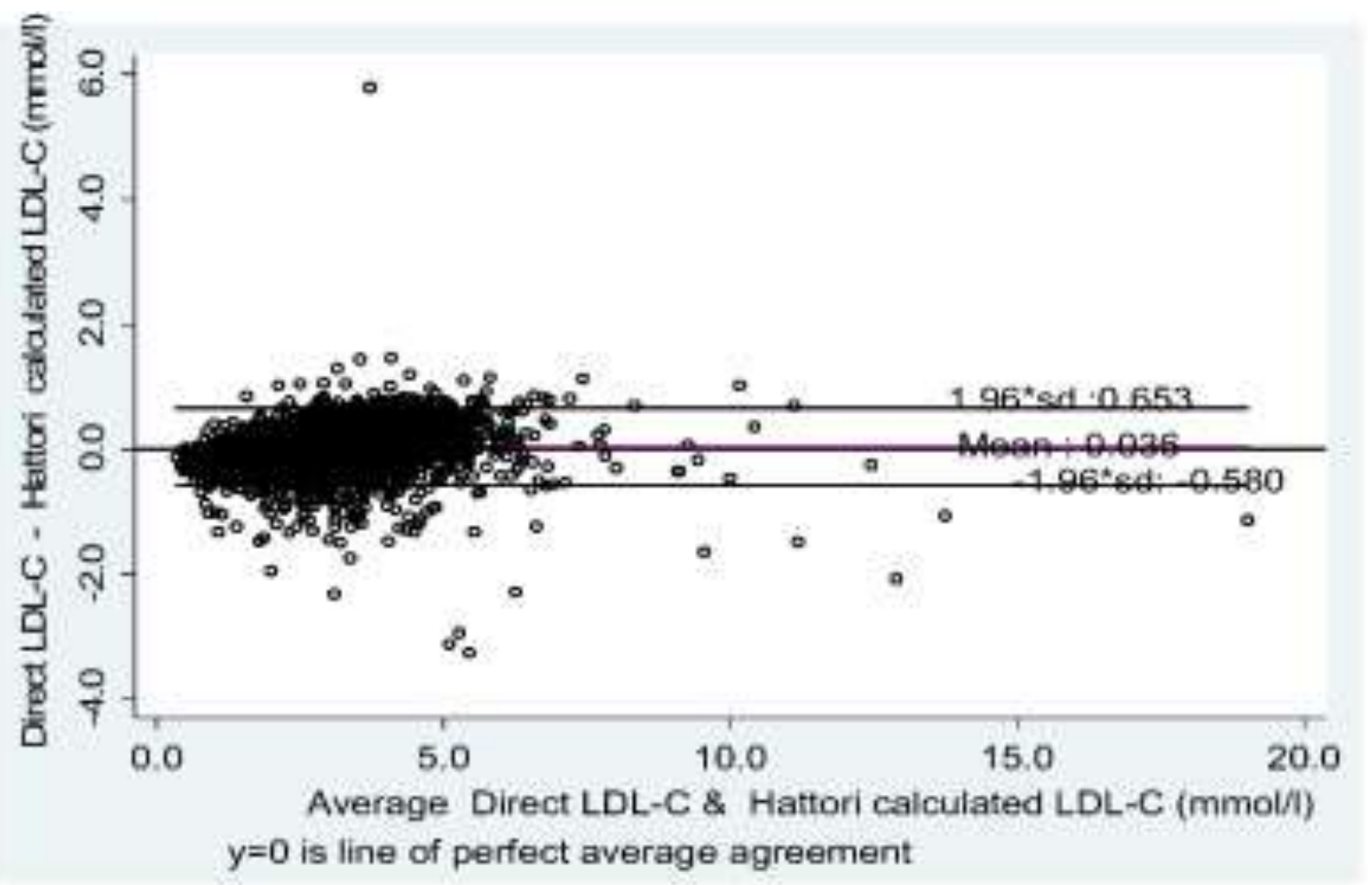

Figure 3 Bland-Altman plots of direct LDL-C and Hattori calculated LDL-C. SI conversion from mmol// to $\mathrm{mg} / \mathrm{dl}$ for LDL-C: $\div 0.0259$

\section{Discussion}

LDL-C concentrations are a primary target of diagnosis and treatment of patients with hyperlipidemia defined by The National Cholesterol Education Program (NCEP) Adult Treatment Panel (ATP) III [1, 2] . LDL-C monitoring remains significant in the management of CVD risk despite the revised AHA practice guidelines which no longer 
support the use of a LDL target [26]. One of the most common problems in the laboratory is to accurately estimate LDL-C. This has important implications on CVD classification, and if done incorrectly can adversely influence therapy and outcomes in patients. We show that the recently published formula by de Cordova et al. to calculate LDL-C correlates highly with direct measurements of LDL-C and is comparable to the Friedewald calculation. However, based on the degree of variation, the Friedewald shows a better agreement with directly measured LDL-C $[27.84 \mathrm{mg} / \mathrm{dl}$ (0.72 mmol/l)]. The de Cordova formula will underestimate an LDL-C by $6.57 \mathrm{mg} / \mathrm{dl}$ (0.17 mmol/l), varying from -30.94 to $44.47 \mathrm{mg} / \mathrm{dl}(-0.80$ to $1.15 \mathrm{mmol} / \mathrm{l})$, with a discrepancy of up to $37.5 \mathrm{mg} / \mathrm{dl}(0.97 \mathrm{mmol} / \mathrm{l})$ for any value of LDL-C, which is higher than previously reported [24]. The Chen formula was the only one to overestimate LDL-C, with a level of discrepancy of $27.84 \mathrm{mg} / \mathrm{dl}(0.72 \mathrm{mmol} / \mathrm{l})$. The Hattori formula will underestimate LDL-C by only $1.55 \mathrm{mg} / \mathrm{dl}(0.04 \mathrm{mmol} / \mathrm{l})$, demonstrating the best agreement with LDL-C with a level of discrepancy of $23.98 \mathrm{mg} / \mathrm{dl}(0.62 \mathrm{mmol} / \mathrm{l})$. We found a similar pattern of negative bias the higher the LDL-C values and a positive bias the lower the LDL-C level using the de Cordova formula, as shown in their study [24]. The other three formulae showed a more uniform distribution of points with the Bland-Altman plots, making the bias observed with these formulae more predictable than the de Cordova formula. We compared the accuracy between four formulae in calculating LDL-C, and found that the Hattori formula performed best across a range of lipid values in a large database of hospitalized patients.

The Friedewald formula is the most widely used to calculate LDL-C. However, there are several other formulae that have been developed in an attempt to address the limitations of the Friedewald formula [10, 15-19]. The limitations of the Friedewald formula include inaccuracy in patients with hypertriglyceridemia [7], in those with very 
low levels of TG $[<100.10 \mathrm{mg} / \mathrm{dl}(<1.13 \mathrm{mmol} / \mathrm{l})][10]$ and $\mathrm{LDL}[<92.81 \mathrm{mg} / \mathrm{dl}(<2.4$ $\mathrm{mmol} / \mathrm{I})$ ] [8], in patients with type III hyperlipidemia[7], in patients with renal [27] and liver [28] disease, and those with diabetes mellitus [11] and other metabolic conditions [29]. The Friedewald formula cannot be used in non-fasting samples as it does not consider the cholesterol formed post-prandially in chylomicrons [30] or in the intermediate-density lipoproteins or in lipoprotein (a) $(\operatorname{Lp}(a))$. The Friedewald formula does not distinguish between cholesterol derived from LDL and $L p(a)$, and thus the LDL-C fraction will be overestimated when $L p(a)$ levels are raised. Furthermore, the Friedewald formula was derived when an LDL-C lower than $70 \mathrm{mg} / \mathrm{dL}(1.8 \mathrm{mmo} / \mathrm{l})$ was not yet established as an ideal secondary prevention target for treatment of high-risk patients [31] [32]; thus these levels were not part of the original data set [7]. The de Cordova formula is the most recently published formula and reports better accuracy than the Friedewald in calculating LDL-C [23]. However, a subsequent analysis of 597 healthy subjects showed better agreement of the Friedewald formula with a directly measured LDL-C [24]. Another recent study compared four formulae in 164 subjects including those with dyslipidemias and co-morbidities and found that the Friedewald equation had the best overall performance for calculating LDL-C [33]. These studies did not compare the Friedewald with the Hattori formula.

In the calculation of LDL-C, three measurements are usually used, including TC, HDLC, and TG. Therefore, the accuracy of calculated LDL-C can be affected by errors from any of these measurements. The aforementioned study also compared calculated LDL-C formulae with 8 directly measured HDL-C assays using homogenous methods and demonstrated that the optimum equation for calculating LDL-C depends on which direct HDL-C assay is used [33]. It was shown that the Daiichi 2-phase method used in our study to measure HDL-C had the third lowest 
percentage misclassifications using the Friedewald formula, and the second lowest with the Chen formula in a recent study comparing eight HDL-C assays. Use of different TC and TG methods is not as likely to significantly affect the calculation of LDL-C as much as direct HDL-C assays because of the better standardization of TC and TG. The TC and TG methods used in our study also differed from their respective reference methods by less than $2 \%$.

We confirm previous findings that the Friedewald formula's performance decreases with increasing TG levels [23], and demonstrate that it performed most poorly at the lowest TG levels. The Friedewald formula was previously shown to incorrectly estimate LDL-C at the lowest TG levels [8, 10], which might have implications for patients on lipid-lowering medications. At the lowest end of TG levels $[17.71 \mathrm{mg} / \mathrm{dl}-$ $90.35 \mathrm{mg} / \mathrm{dl}(0.20-1.02 \mathrm{mmol} / \mathrm{l})]$, the de Cordova formula had the highest accuracy, contrary to a similar analysis in healthy subjects [24]. The differences may be attributed to the different methods of measuring LDL-C and HDL-C between those two studies (Wako vs Daiichi). However, our study findings are similar to those of de Cordova, despite our different methods of measuring LDL-C and HDL-C. The de Cordova study used a large Brazlian cohort including healthy persons and patients with hyperlipidemia and diabetes. For this reason, the de Cordova formula was suitable to be validated within a diverse hospital population such as ours. The similarities of our findings to those of de Cordova might be due to the populations studied, despite the different methods used. Although the de Cordova formula does not require fasting samples as it does not consider TG levels, it performed least well in the hypertriglyercidaemic samples. At the highest level of TG [>187 mg/dl $(>2.11$ $\mathrm{mmol} / \mathrm{l})$ ], the Hattori formula outperformed all other three formula by a difference of more than 200 rMSE, not seen in the large Brazilian database (22). This finding 
should take into consideration that the Hattori formula was not validated in subjects with TG $[<30 \mathrm{mg} / \mathrm{dl}(<0.34 \mathrm{mmol} / \mathrm{l})]$ and $[>400.4 \mathrm{mg} / \mathrm{dl}(>4.52 \mathrm{mmol} / \mathrm{l})][17]$. In contrast to the de Cordova study, the Chen formula outperformed the de Cordova formula at low TG levels in addition to high TG levels. Chen's formula includes TG values for the calculation of LDL-C [16], so the differences in comparison to other formulae at the extremities of TG are to be expected. Furthermore, methods to measure HDL-C used in the Chen formula (Roche) have fewer misclassifications than the method used by de Cordova (Wako) [33].

The limitations of our study include the fact that racial origins were not specified and could not be considered in the analysis. However, the database is from a large hospitalized population representative of the various ethnic origins in South Africa. Secondly, although we used a non-reference method for the measurement of LDL-C, as with two similar previous analyses $[23,24]$, our methods meet the NCEP standards of precision. Although we were unable to evaluate the formulae by disease categories, we had a large sample size with varying lipid ranges. We were thus able to consider the effect of the four formulae at high and low TG values, similarly to the de Cordova study. Another limitation is that we focused only on four LDL-C calculations, using the most recently published [23], most widely used [7] and two formulae previously confirmed in a large database to perform well in extreme lipid values [16, 17, 23], as seen in our hospitalized study sample. The samples we analyzed were from hospitalized patients and the findings cannot be generalized to the general population. However, we do report these analyses on the largest database to date - 14,219 patients. Although patient-specific data about presence/absence of disease, treatments and ethnicity was not available, our database of hospitalized patients is 
representative of those with diabetes, dyslipidemia and other metabolic conditions and co-morbidities.

Formulae have reported poorer performance in low- and high TG values, and it has been suggested to use direct measurements of LDL-C instead of calculations in hypertriglyceridaemic samples $[4,33]$. Measurements of LDL-C are further complicated by LDL-C being a multiple molecular particle aggregate of protein, cholesterol and other lipids [34]. Normal LDL-C is often observed in myocardial infarction, but with increased LDL-apolipoprotein B (Apo B) $[17,35]$. It is these small dense LDL particles that are more highly correlated with CVD, rather than the concentration of particles present [36]. The contribution of these aggregates is not fully considered in the existing formulae to calculate LDL-C. In a prospective study of 2222 men free from ischaemic heart disease (IHD), correction of the Friedewald formula to account for $L p(a)$ levels (the Dahlen modification) did not improve the evaluation of IHD risk [37]. One recent study considered the variance in the TG:Very low density lipoprotein cholesterol (VLDL-C) ratio, and found that using a 180 panel specific to TG and HDL-C levels improves the accuracy of their formula (non-HDL-C TG/adjustable factor $\mathrm{mg} / \mathrm{dL}$ ) as compared to Friedewald [21]. However, a subsequent validation study found uncertainties in both this novel formula and Friedewald at low LDL-C levels (<1.18 mmol/I) [22]. Accuracy of the formulae may be improved where TG/VLDL-C ratios are taken into consideration [21], particularly in hypertriglyceridaemic patients. The authors found that most of the variance in the ratios could be explained by TG and non-HDL-C levels. The latter observation could further explain the differences in performance of the formulae, as the Chen formula equates LDL-C to $90 \%$ of non-HDL-C plus $10 \%$ of triglycerides, whereas de Cordova takes $75 \%$ of non-HDL-C and Hattori $94 \%$ of non-HDL-C. 
Debate thus exists on whether two alternative markers - non-HDL-C (the sum of masses of cholesterol in the Apo B lipoprotein particles) and Apo B (the main apoprotein of atherogenic lipoproteins) should supplant LDL-C in CVD risk calculations. At present, there exists insufficient evidence to warrant this substitution [38]. However, the markers may provide additive value to CVD risk assessment [39]. We have demonstrated that LDL-C and TC correlate highly with non-HDL-C, as shown previously [23]. Recent reviews have established the superiority of non-HDL-C and ApoB over LDL-C in predicting CVD risk in epidemiological studies[35] and in randomized trials of patients on statin treatment[40]. Non-HDL-C has been recommended by previous ATP III guidelines as a secondary target of therapy and to be used to assess risk in patients with elevated TG levels [>200 mg/dl $(>2.26 \mathrm{mmol} / \mathrm{l})]$ $[1,2]$, with the latter confirmed by a subsequent study comparing non-HDL-C to direct and calculated LDL-C using 8 different assays [4]. In terms of clinical practice, revised guidelines by the AHA report no additional mortality benefit to further treat non-HDL-C levels once an LDL-C goal is reached [26]. Concerns also exist about the reliability of non-HDL-C measurements, as a result of problems with direct HDL-C measurements [29]. Alternatively, Apo B and its association with CVD risk have been recognized [39, 40], reportedly performing better than LDL-C in hypertriglyercedaemic patients [35, 41] and as an index of LDL-lowering therapy [39]. The Hattori formula for LDL-C (0.94TC - 0.94HDL-C - $0.19 \times$ TG) was developed to estimate LDL-Apo B and small dense LDL from blood cholesterol, TG, HDL-C and in this way be more accurate in patients with cardiovascular co-morbidities and dyslipidemias. Unlike the Friedewald formula, the Hattori formula excludes IDL to provide a more accurate estimate of LDL-C. The estimation of lipid particles in the Hattori formula may explain why that particular formula performs best across various TG, HDL and TC levels in our hospitalized 
population. Formulae that incorporate Apo B or non-HDL-C measurements may be of interest in pursuing LDL-C calculations to predict CVD risk.

\section{Conclusions}

In conclusion, we confirm recent findings that the Friedewald formula has a better agreement with directly measured LDL-C based on the Daiichi method compared with the agreement with the de Cordova [23], but not at low TG values in a large hospitalized population. Furthermore, we show that neither the Friedewald or de Cordova formula perform as well as the Chen or Hattori formula in this population group.

\section{Competing interests}

The authors have no conflicts of interest.

\section{Acknowledgements}

Barbara English for proofreading of the manuscript.

NHLS for access to the database

\section{References}

[1] Expert Panel on Detection E, Treatment of High Blood Cholesterol in A. Executive Summary of The Third Report of The National Cholesterol Education Program (NCEP) Expert Panel on Detection, Evaluation, And Treatment of High Blood Cholesterol In Adults (Adult Treatment Panel III). JAMA : the journal of the American Medical Association 2001; 285:2486-2497.

[2] National Cholesterol Education Program Expert Panel on Detection E, Treatment of High Blood Cholesterol in A. Third Report of the National Cholesterol Education Program (NCEP) Expert Panel on Detection, Evaluation, and Treatment of High Blood Cholesterol in Adults (Adult Treatment Panel III) final report. Circulation 2002; 106:3143-3421.

[3] Dong J, Guo H, Yang R, et al. Serum LDL- and HDL-cholesterol determined by ultracentrifugation and HPLC. Journal of lipid research 2011; 52:383-388.

[4] van Deventer HE, Miller WG, Myers GL, et al. Non-HDL cholesterol shows improved accuracy for cardiovascular risk score classification compared to direct or calculated LDL cholesterol in a dyslipidemic population. Clinical chemistry 2011; 57:490-501.

[5] Miller WG, Myers GL, Sakurabayashi I, et al. Seven direct methods for measuring HDL and LDL cholesterol compared with ultracentrifugation reference measurement procedures. Clinical chemistry 2010; 56:977-986.

[6] Nauck M, Warnick GR, Rifai N. Methods for measurement of LDL-cholesterol: a critical assessment of direct measurement by homogeneous assays versus calculation. Clinical chemistry 2002; 48:236-254. 
[7] Friedewald WT, Levy RI, Fredrickson DS. Estimation of the concentration of low-density lipoprotein cholesterol in plasma, without use of the preparative ultracentrifuge. Clinical chemistry 1972; 18:499-502.

[8] Scharnagl $\mathrm{H}$, Nauck M, Wieland $\mathrm{H}$, Marz W. The Friedewald formula underestimates LDL cholesterol at low concentrations. Clinical chemistry and laboratory medicine : CCLM / FESCC 2001; 39:426-431.

[9] de Cordova CM, Schneider CR, Juttel ID, de Cordova MM. Comparison of LDL-cholesterol direct measurement with the estimate using the Friedewald formula in a sample of 10,664 patients. Arquivos brasileiros de cardiologia 2004; 83:482-487; 476-481.

[10] Ahmadi SA, Boroumand MA, Gohari-Moghaddam K, Tajik P, Dibaj SM. The impact of low serum triglyceride on LDL-cholesterol estimation. Archives of Iranian medicine 2008; 11:318-321.

[11] Rubies-Prat J, Reverter JL, Senti M, et al. Calculated low-density lipoprotein cholesterol should not be used for management of lipoprotein abnormalities in patients with diabetes mellitus. Diabetes care 1993; 16:1081-1086.

[12] Gazi I, Tsimihodimos V, Filippatos TD, et al. LDL cholesterol estimation in patients with the metabolic syndrome. Lipids in health and disease 2006; 5:8.

[13] Gazi IF, Elisaf M. LDL-cholesterol calculation formulas in patients with or without the metabolic syndrome. International journal of cardiology 2007; 119:414-415.

[14] Vohnout B, Vachulova A, Blazicek P, Dukat A, Fodor G, Lietava J. Evaluation of alternative calculation methods for determining LDL cholesterol. Vnitrni lekarstvi 2008; 54:961-964.

[15] Anandaraja S, Narang R, Godeswar R, Laksmy R, Talwar KK. Low-density lipoprotein cholesterol estimation by a new formula in Indian population. International journal of cardiology 2005; 102:117-120.

[16] Chen $\mathrm{Y}$, Zhang $\mathrm{X}$, Pan B, et al. A modified formula for calculating low-density lipoprotein cholesterol values. Lipids in health and disease 2010; 9:52.

[17] Hattori Y, Suzuki M, Tsushima M, et al. Development of approximate formula for LDL-chol, LDL-apo B and LDL-chol/LDL-apo B as indices of hyperapobetalipoproteinemia and small dense LDL. Atherosclerosis 1998; 138:289-299.

[18] Vujovic A, Kotur-Stevuljevic J, Spasic S, et al. Evaluation of different formulas for LDL-C calculation. Lipids in health and disease 2010; 9:27.

[19] Teerakanchana T, Puavilai W, Suriyaprom K, Tungtrongchitr R. Comparative study of LDLcholesterol levels in Thai patients by the direct method and using the Friedewald formula. The Southeast Asian journal of tropical medicine and public health 2007; 38:519-527.

[20] Rao A, Parker AH, el-Sheroni NA, Babelly MM. Calculation of low-density lipoprotein cholesterol with use of triglyceride/cholesterol ratios in lipoproteins compared with other calculation methods. Clinical chemistry 1988; 34:2532-2534.

[21] Martin SS, Blaha MJ, Elshazly MB, et al. Comparison of a novel method vs the Friedewald equation for estimating low-density lipoprotein cholesterol levels from the standard lipid profile. JAMA : the journal of the American Medical Association 2013; 310:2061-2068.

[22] Meeusen JW, Lueke AJ, Jaffe AS, Saenger AK. Validation of a Proposed Novel Equation for Estimating LDL Cholesterol. Clinical chemistry 2014; 60:1519-1523.

[23] de Cordova CM, de Cordova MM. A new accurate, simple formula for LDL-cholesterol estimation based on directly measured blood lipids from a large cohort. Annals of clinical biochemistry 2013; 50:13-19.

[24] Onyenekwu CP, Hoffmann M, Smit F, Matsha TE, Erasmus RT. Comparison of LDL-cholesterol estimate using the Friedewald formula and the newly proposed de Cordova formula with a directly measured LDL-cholesterol in a healthy South African population. Annals of clinical biochemistry 2014.

[25] Nakamura M, Tanigut Y, Yamamoto M, Hino K, Manabe M. Homogenous assay of serum LDL Cholesterol on an auto analyzer. Clinical chemistry 1997; 43:S260-S261.

[26] Stone NJ, Robinson JG, Lichtenstein AH, et al. 2013 ACC/AHA Guideline on the Treatment of Blood Cholesterol to Reduce Atherosclerotic Cardiovascular Risk in Adults: A Report of the American College of Cardiology/American Heart Association Task Force on Practice Guidelines. Circulation 2014; 129:S1-S45.

[27] Nauck M, Kramer-Guth A, Bartens W, Marz W, Wieland H, Wanner C. Is the determination of LDL cholesterol according to Friedewald accurate in CAPD and HD patients? Clinical nephrology 1996; 46:319-325.

[28] Matas C, Cabre M, La Ville A, et al. Limitations of the Friedewald formula for estimating lowdensity lipoprotein cholesterol in alcoholics with liver disease. Clinical chemistry 1994; 40:404-406. 
[29] McNamara JR, Cohn JS, Wilson PW, Schaefer EJ. Calculated values for low-density lipoprotein cholesterol in the assessment of lipid abnormalities and coronary disease risk. Clinical chemistry 1990; 36:36-42.

[30] Contois JH, Warnick GR, Sniderman AD. Reliability of low-density lipoprotein cholesterol, nonhigh-density lipoprotein cholesterol, and apolipoprotein B measurement. Journal of clinical lipidology 2011; 5:264-272.

[31] Grundy SM, Cleeman JI, Merz CN, et al. Implications of recent clinical trials for the National Cholesterol Education Program Adult Treatment Panel III Guidelines. Journal of the American College of Cardiology 2004; 44:720-732.

[32] European Association for Cardiovascular P, Rehabilitation, Reiner Z, et al. ESC/EAS Guidelines for the management of dyslipidaemias: the Task Force for the management of dyslipidaemias of the European Society of Cardiology (ESC) and the European Atherosclerosis Society (EAS). European heart journal 2011; 32:1769-1818.

[33] Oliveira MJ, van Deventer HE, Bachmann LM, et al. Evaluation of four different equations for calculating LDL-C with eight different direct HDL-C assays. Clinica chimica acta; international journal of clinical chemistry 2013; 423:135-140.

[34] Cromwell WC, Otvos JD, Keyes MJ, et al. LDL Particle Number and Risk of Future Cardiovascular Disease in the Framingham Offspring Study - Implications for LDL Management. Journal of clinical lipidology 2007; 1:583-592.

[35] Sniderman AD, Williams $\mathrm{K}$, Contois $\mathrm{JH}$, et al. A meta-analysis of low-density lipoprotein cholesterol, non-high-density lipoprotein cholesterol, and apolipoprotein B as markers of cardiovascular risk. Circulation Cardiovascular quality and outcomes 2011; 4:337-345.

[36] Superko HR, Gadesam RR. Is it LDL particle size or number that correlates with risk for cardiovascular disease? Current atherosclerosis reports 2008; 10:377-385.

[37] Cantin B, Lamarche B, Despres JP, Dagenais GR. Does correction of the friedewald formula using lipoprotein(a) change our estimation of ischemic heart disease risk? The Quebec Cardiovascular Study. Atherosclerosis 2002; 163:261-267.

[38] Goff DC, Jr., Lloyd-Jones DM, Bennett G, et al. 2013 ACC/AHA Guideline on the Assessment of Cardiovascular Risk: A Report of the American College of Cardiology/American Heart Association Task Force on Practice Guidelines. Circulation 2014; 129:S49-73.

[39] Perk J, De Backer G, Gohlke H, et al. European Guidelines on cardiovascular disease prevention in clinical practice (version 2012). The Fifth Joint Task Force of the European Society of Cardiology and Other Societies on Cardiovascular Disease Prevention in Clinical Practice (constituted by representatives of nine societies and by invited experts). European heart journal 2012; 33:16351701.

[40] Contois JH, McConnell JP, Sethi AA, et al. Apolipoprotein B and cardiovascular disease risk: position statement from the AACC Lipoproteins and Vascular Diseases Division Working Group on Best Practices. Clinical chemistry 2009; 55:407-419.

[41] Boekholdt SM, Arsenault BJ, Mora S, et al. Association of LDL cholesterol, non-HDL cholesterol, and apolipoprotein $B$ levels with risk of cardiovascular events among patients treated with statins: a meta-analysis. JAMA : the journal of the American Medical Association 2012; 307:1302-1309. 


\section{Supplementary Figures}

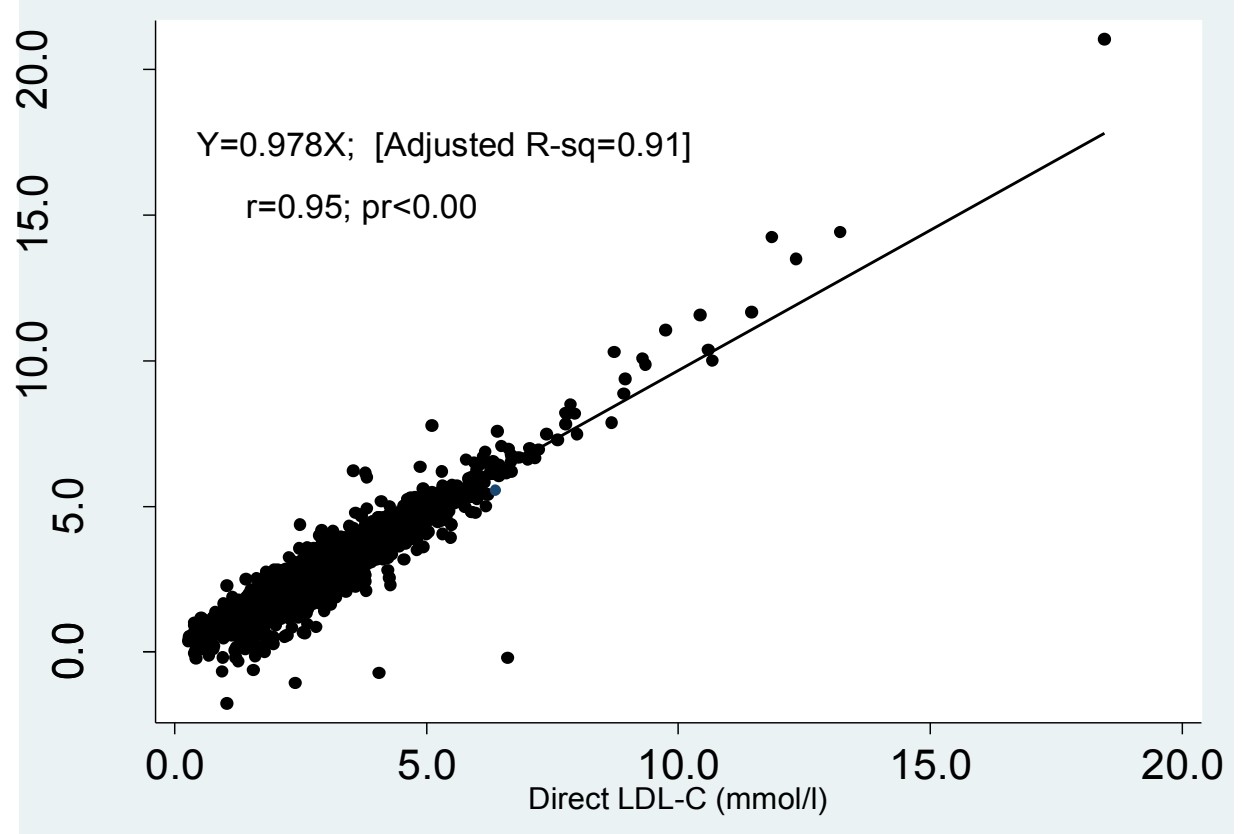

Supplementary Figure 1A

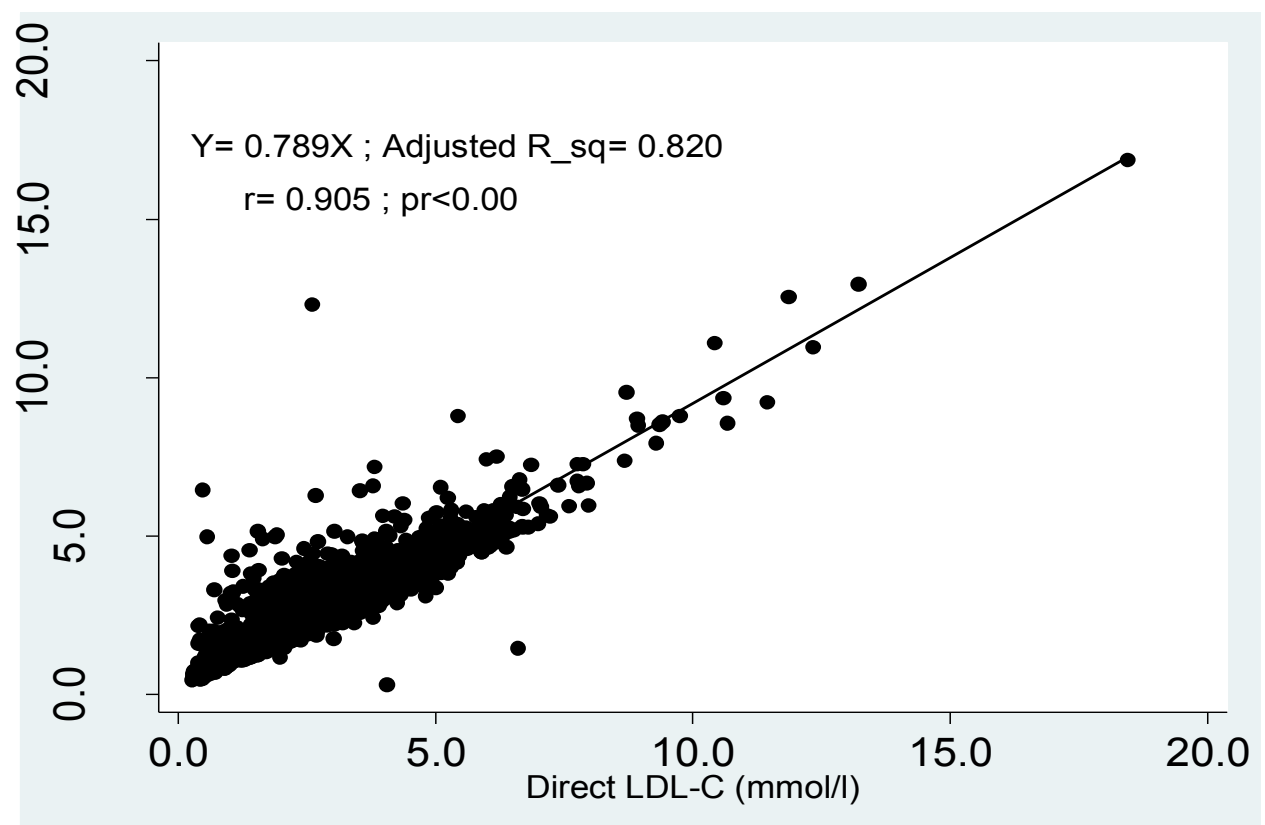

Supplementary Figure 1B 


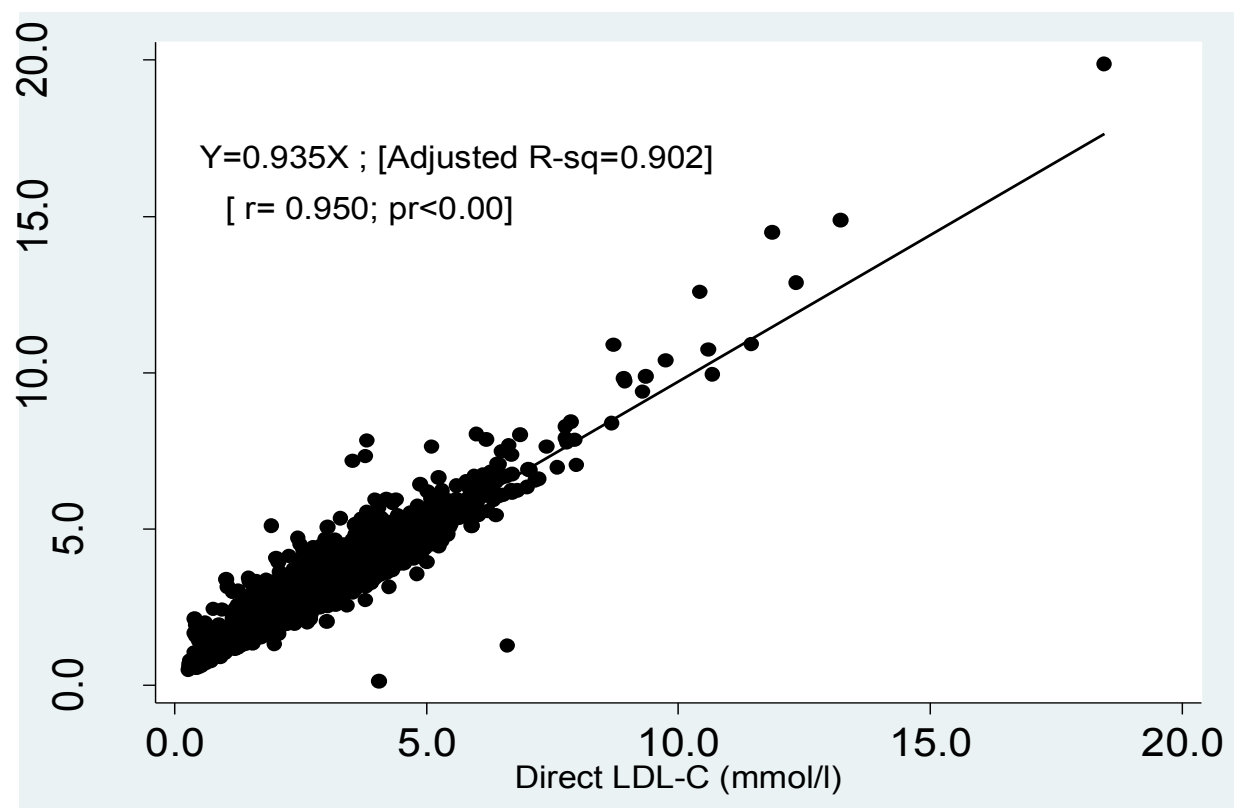

Supplementary Figure 1C

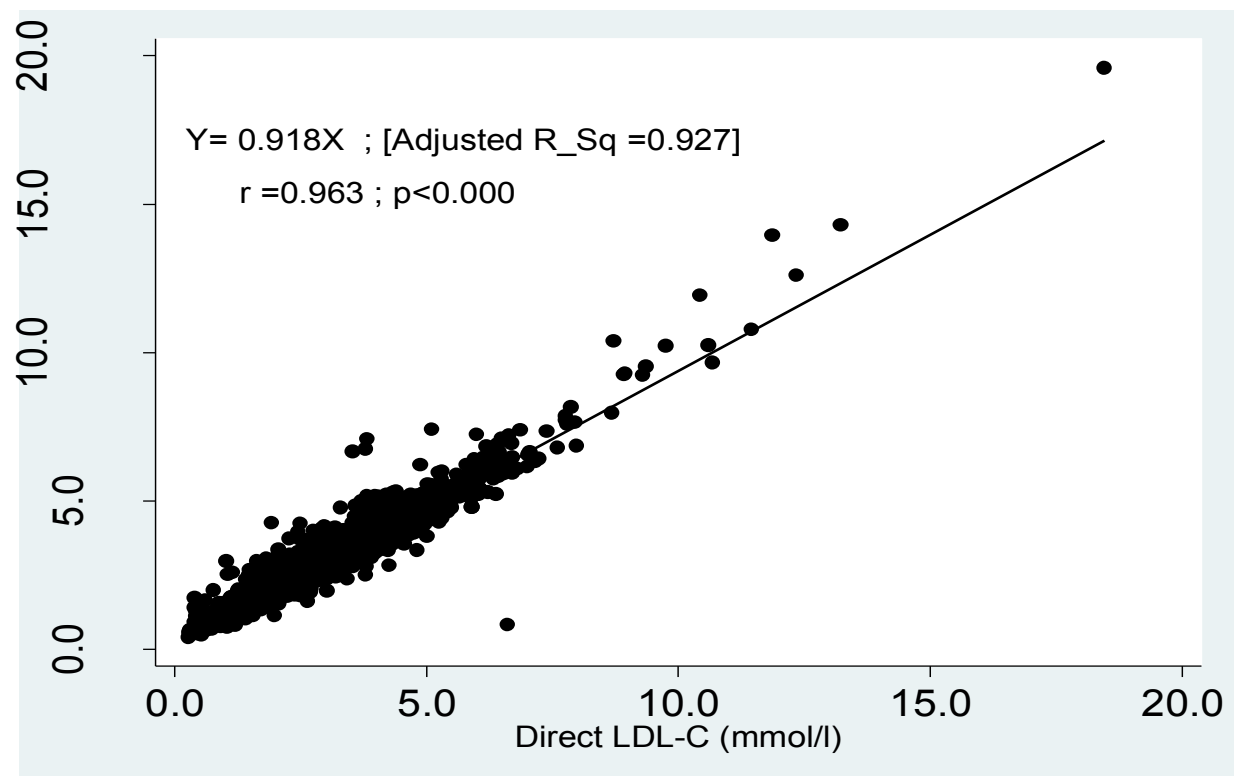

\section{Supplementary Figure 1D}

Supplementary Figure 1. Correlation between the calculated LDL-C by A) the Friedewald formula, B) the de Cordova formula, C) the Chen formula and D) the Hattori formula and directly measured LDL-C. 


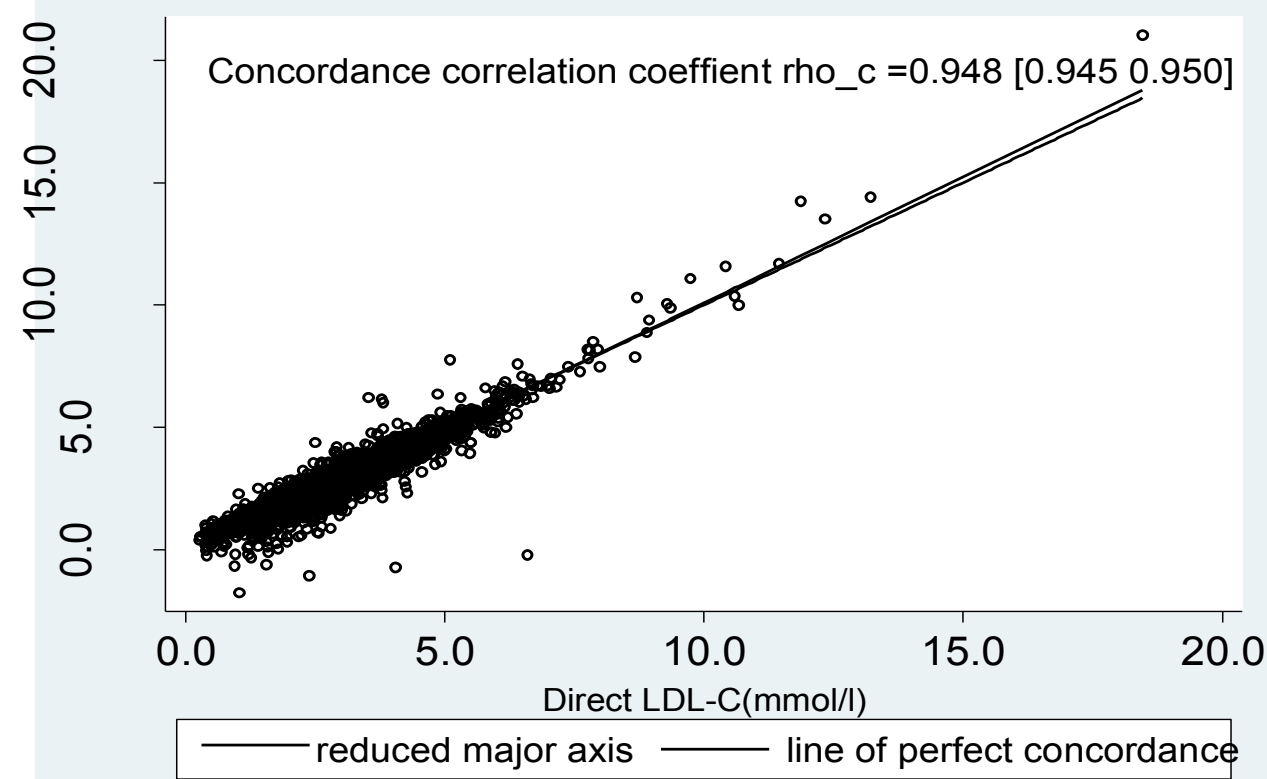

\section{Supplementary Figure 2A}

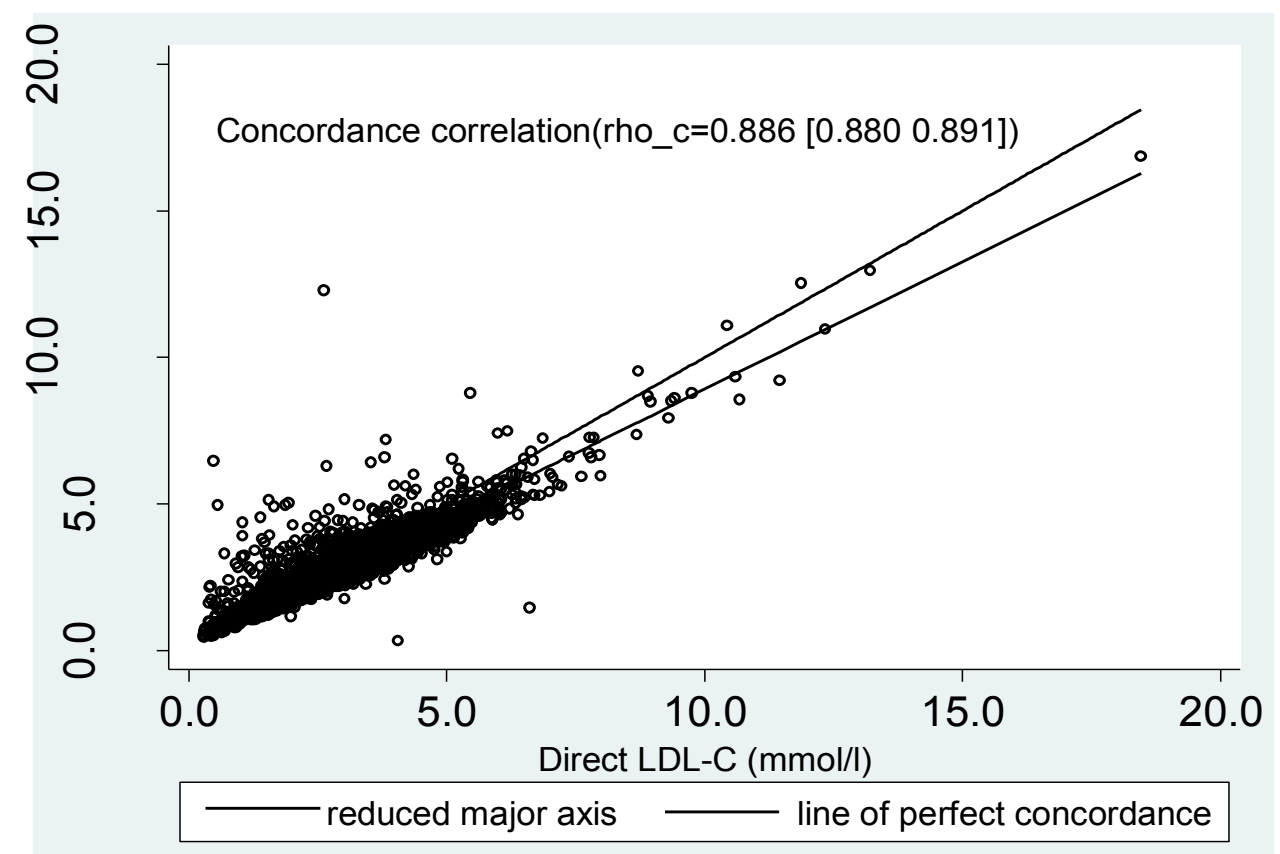

Supplementary Figure 2B 


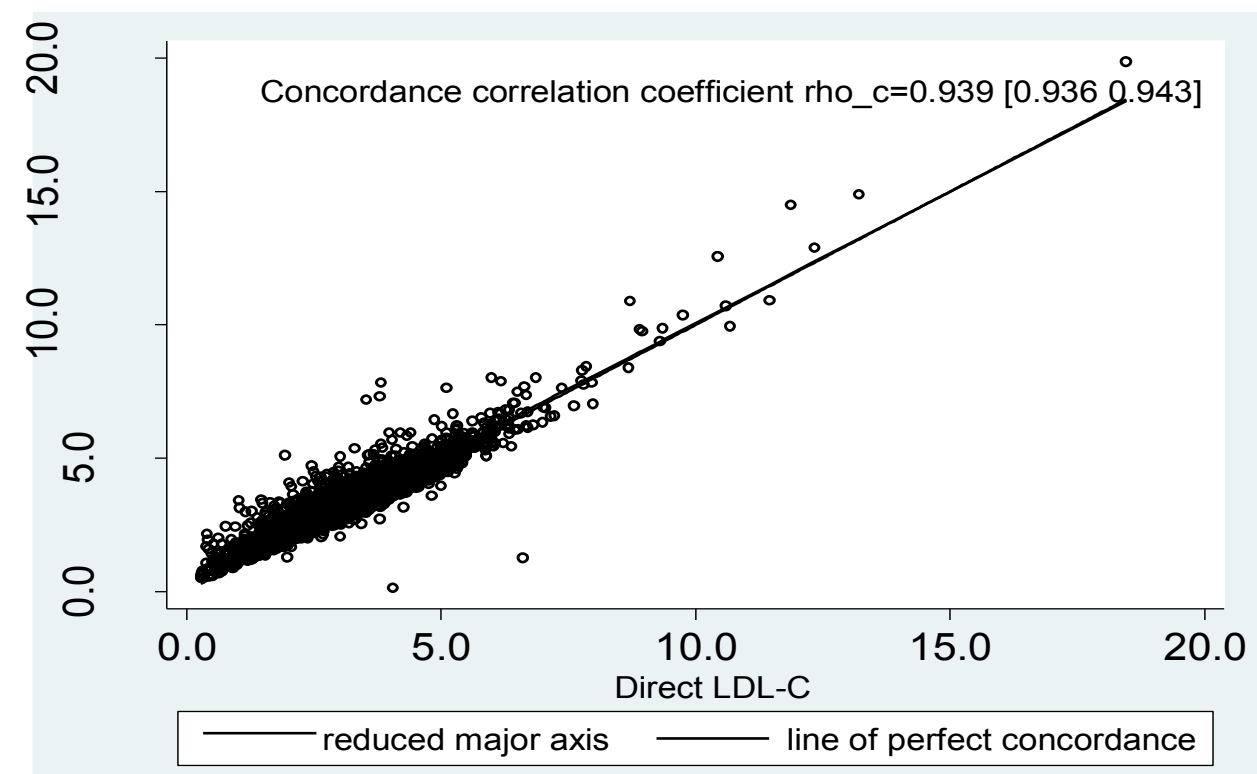

Supplementary figure 2C

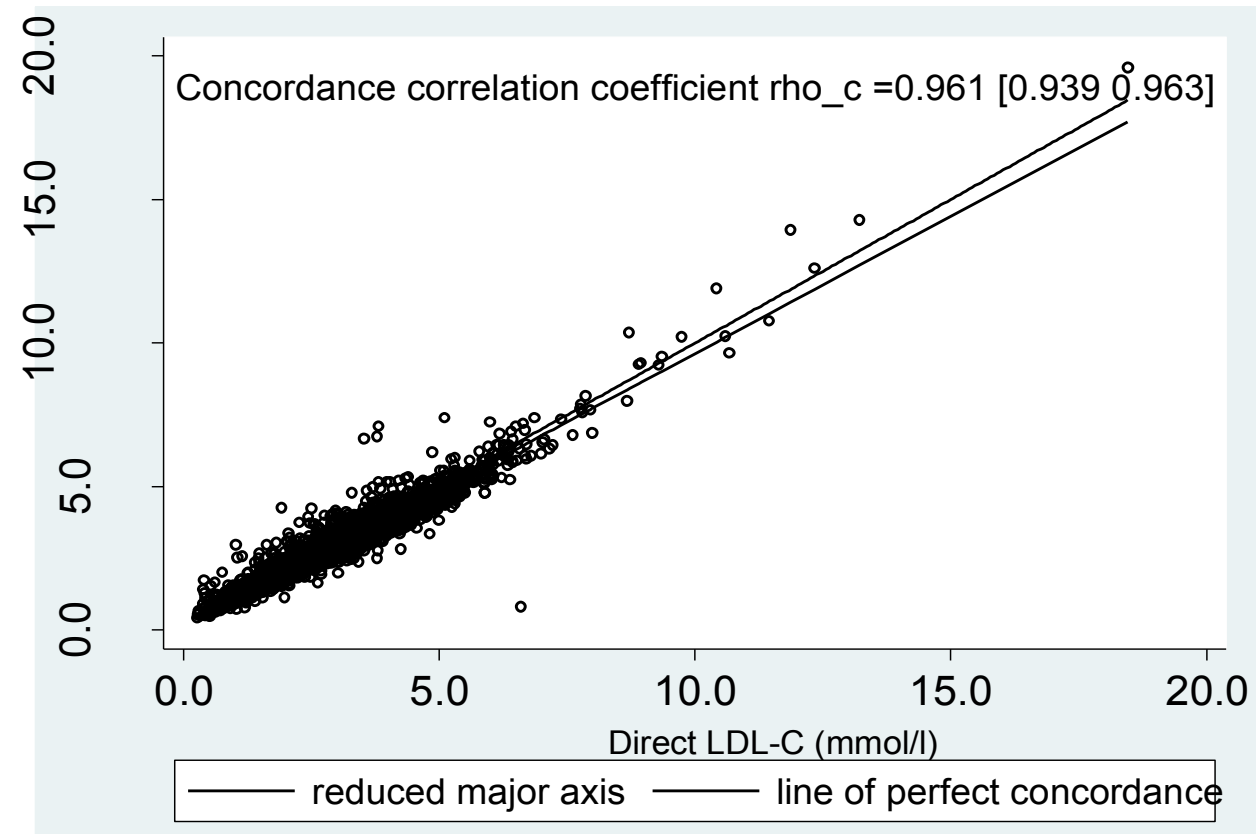

\section{Supplementary figure 2D}

Supplementary Figure 2. Concordance correlation between the calculated LDL-C by the formulae and directly measured LDL-C; A) Friedewald; B) de Cordova; C) Chen; D) Hattori. 


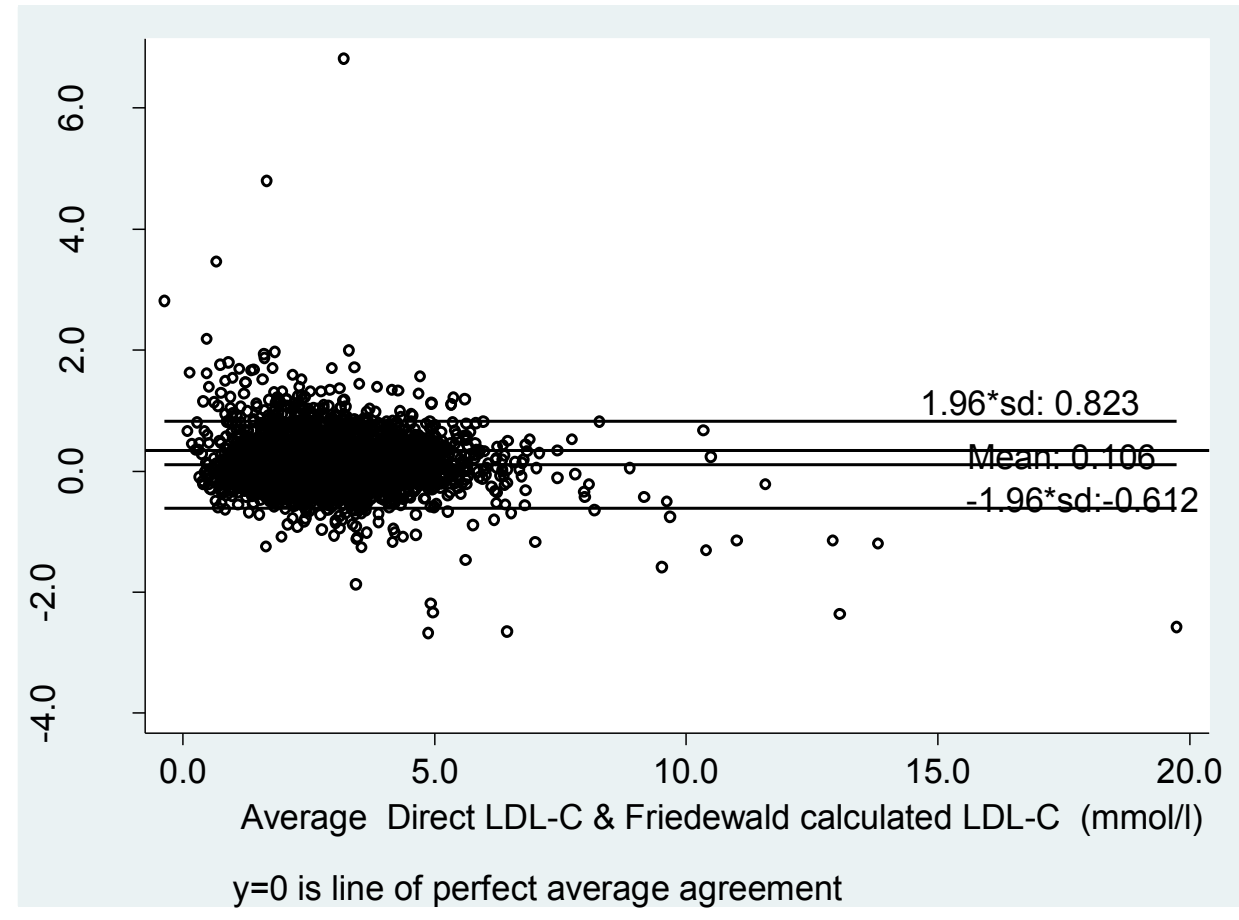

Supplementary Figure 3A

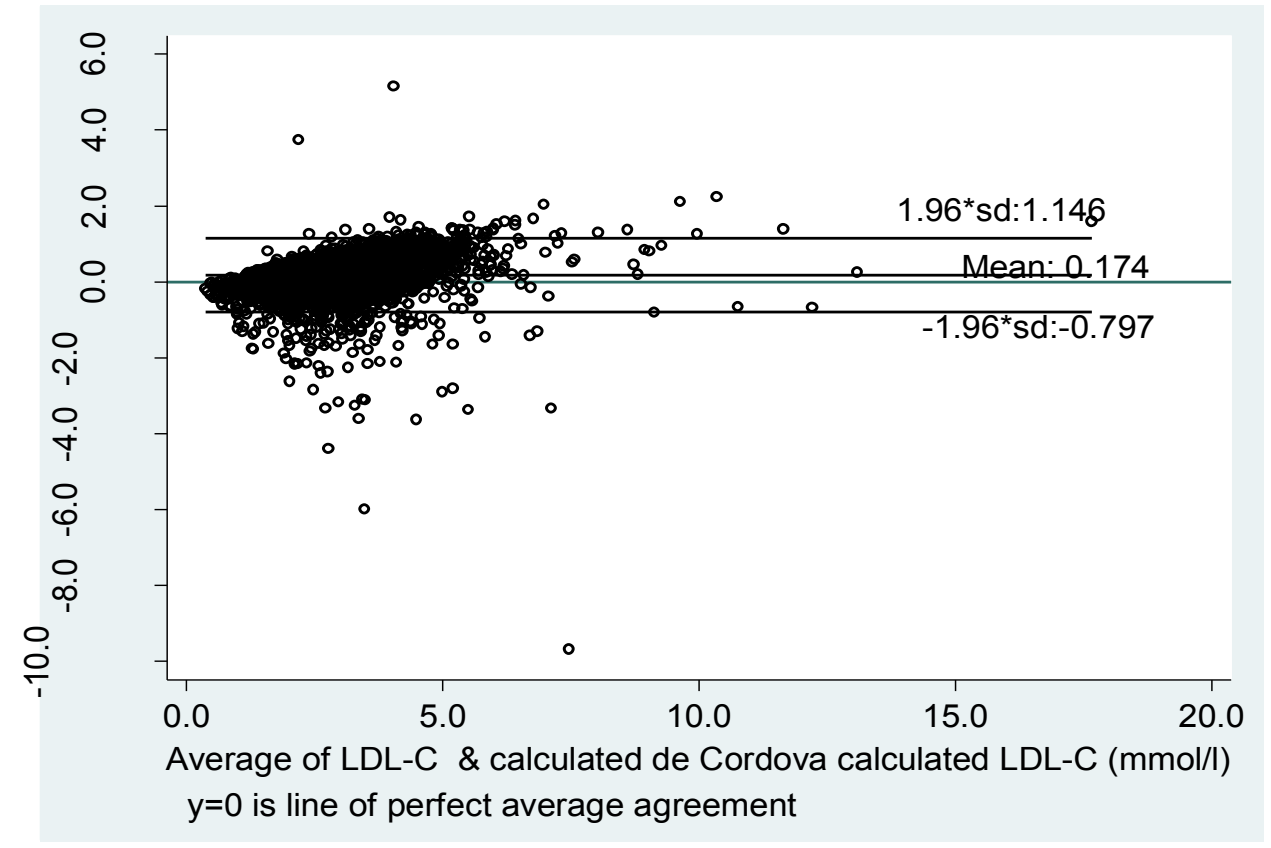

\section{Supplementary Figure 3B}




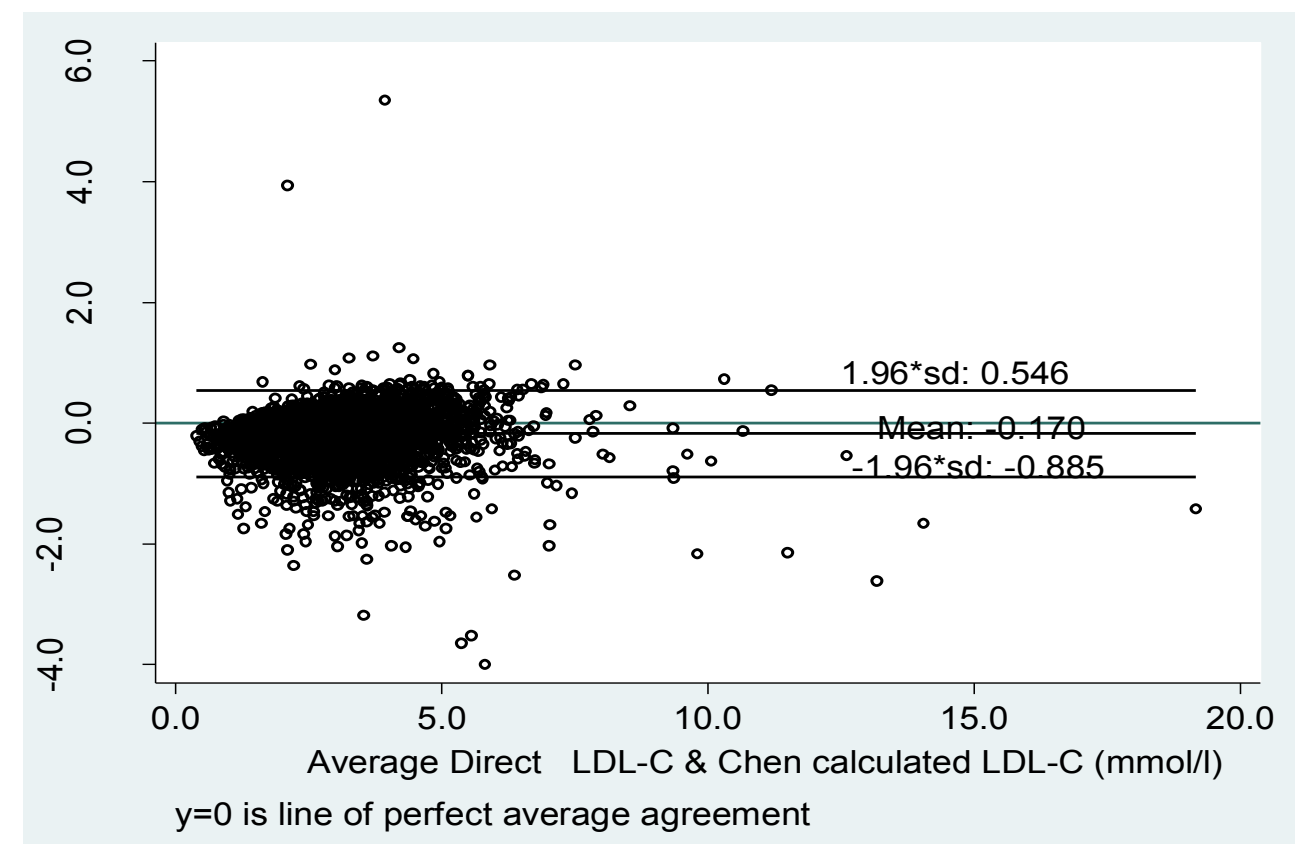

\section{Supplementary Figure $3 \mathrm{C}$}

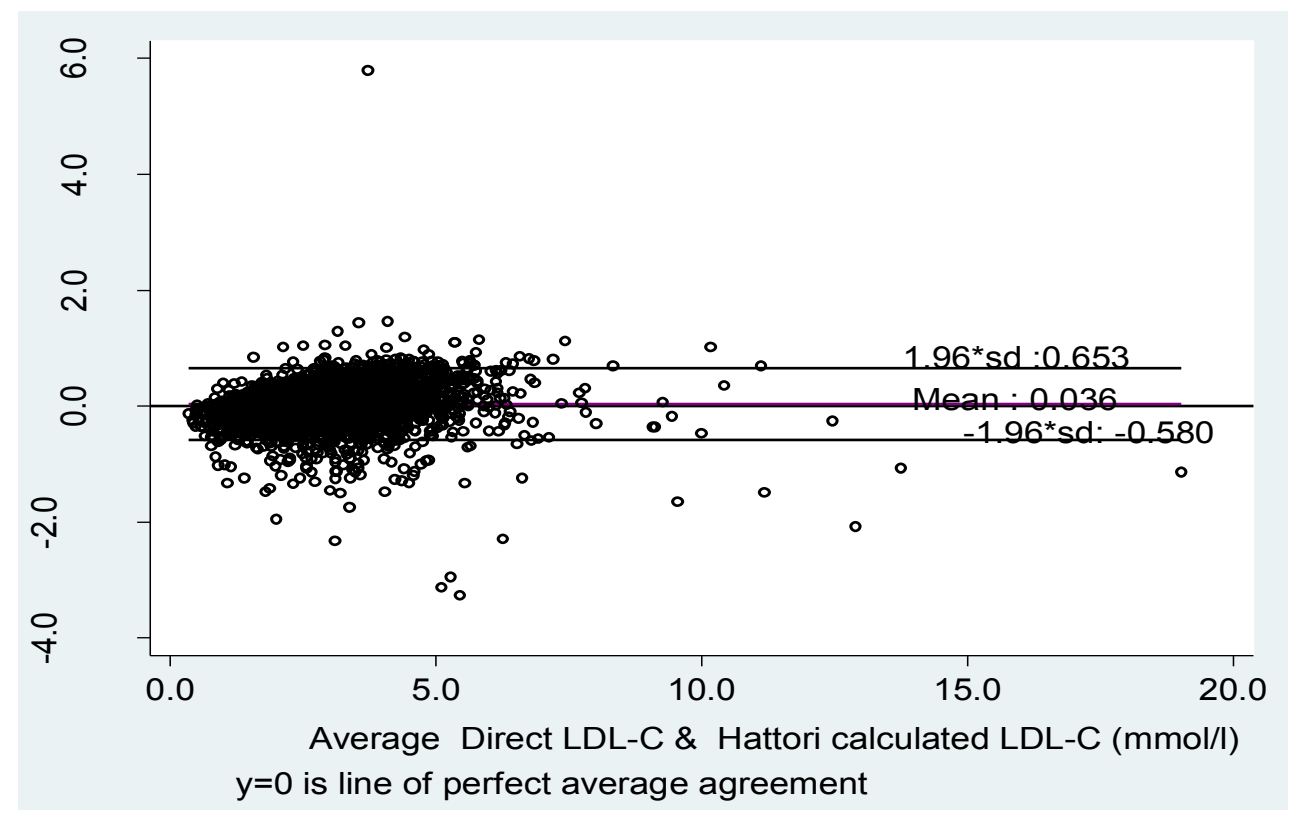

\section{Supplementary Figure 3}

Supplementary Figure 3. Bland-Altman plots of direct LDL-C and A) Friedewald calculated LDL-C, B) de Cordova calculated LDL-C, C) Chen calculated LDL-C and D) Hattori calculated LDL-C. SI conversion from $\mathrm{mmol} / \mathrm{l}$ to $\mathrm{mg} / \mathrm{dl}$ for LDL-C: $\div 0.0259$. 